\title{
The influence of general self-efficacy on the interpretation of vicarious experience information within online learning
}

Natalie Wilde ${ }^{*}$ and Anne Hsu * Correspondence: n.wilde@qmul.ac.
uk
Queen Mary University of London,
Mile End Road, London E1 4NS, UK

\begin{abstract}
An individual's general self-efficacy affects their cognitive behaviours in a number of ways. Previous research has found general self-efficacy to influence how people interpret persuasive messages designed to encourage behavioural change. No previous work has looked into how general self-efficacy affects the interpretation of vicarious experience information and how this affects self-efficacy in being able to complete a set task within a career skills online learning environment. The study presented considers this gap in knowledge, analysing the effect of six different types of vicarious experience information on the self-efficacy of online workshop participants to complete a set task. In analysing the results, each participant's general self-efficacy was considered.

Results showed individuals with low general self-efficacy to find vicarious experience information significantly less beneficial for their self-efficacy in completing a set task when compared to others with high general self-efficacy. Those with low general self-efficacy were more likely to make negative self-comparisons to the vicarious experience information, restricting its potential to increase their self-efficacy. In contrast, participants with high general self-efficacy found many of the vicarious experience information presented to be beneficial to their self-efficacy to complete the set task as they were more likely to dismiss any information they interpreted to be negative. Results from this study highlight the importance of more research into how vicarious experience information can be designed and presented in a way that ensures benefit to the taskspecific self-efficacy of all individuals, regardless of their general self-efficacy beliefs at the time.
\end{abstract}

Keywords: Self-efficacy, Online learning environments, E-learning, Vicarious experience information

\section{Introduction}

Self-efficacy (SE) refers to an individual's belief that they are able to succeed given any task that they encounter (Bandura, 1977). SE can be general or task specific, allowing individuals to have a range of SE beliefs about themselves at any one time. An individual's beliefs surrounding their own levels of SE can have an impact on how they feel, think and motivate themselves. This can lead to significant contrasts in behaviour between individuals with differing levels of SE. Those with a strong or high sense of SE believe in their own capability deeply, seeing challenges as tasks to be mastered rather than threats to be avoided (Bandura, 1977). They also engross themselves into tasks and exert strong

(c) The Author(s). 2019 Open Access This article is distributed under the terms of the Creative Commons Attribution 4.0 International License (http://creativecommons.org/licenses/by/4.0/), which permits unrestricted use, distribution, and reproduction in any medium, provided you give appropriate credit to the original author(s) and the source, provide a link to the Creative Commons license, and indicate if changes were made. 
commitment. Any setbacks they encounter are easily recovered and learned from. These factors can all lead to enhanced personal well being by reducing stress, resulting in the individual being less likely to experience depression. Others with a weak or low sense of SE have major doubts over their own capabilities (Bandura, 1977). This can lead to a total avoidance of challenges as they see them as threatening situations. These individuals can spend a lot of time focussing on their previous failings and this can lead to setbacks being difficult to recover from. For this reason, these individuals can be more vulnerable to depression and stress (Bandura, 1977).

Levels of SE are not static and have the ability to be increased through exposure to influential information sources, one of which is vicarious experience information (VEI). VEI is argued by Gist and Mitchell (1992) to have the most instant and direct effect on an individual's SE. If we read information about someone which implies that they have succeeded at a certain task, it raises our own belief that we too can succeed at the same task. This belief is further increased if we observe an individual that we consider to be similar to ourselves (Schunk, 1987). Previous studies have not considered how an individual's level of general SE affects how they interpret VEI and the benefit to their taskspecific SE that they get from this information.

Bandura (1994) explains how an individual's current general SE can shape their behaviours and this may influence how they interpret and perceive information (Gist \& Mitchell, 1992), which could include VEI. Due to individuals with low general SE being more likely to be brought down by information and to dwell upon previous negative experiences, they may not interpret VEI or pay it as much attention when compared to others with higher levels of general SE (Bandura, 1994). This puts them at a disadvantage, as this may lead to low general SE individuals not finding the VEI as beneficial on their SE. The study presented in this paper considers how an individual's general SE influences how they interpret VEI and the effect this information has on their taskspecific SE. This effect is considered within the context of a career skills online learning environment (OLE). In this study an OLE is defined as 'a virtual environment that allows individuals to access learning material' (Ally, 2004).

The study works with two types of SE beliefs, general and task-specific SE, with distinct differences between the two. General SE beliefs mirror the definition provided by Bandura (1977), 'the belief in one's capabilities to organize and execute the courses of action required to manage prospective situations'. General SE concerns an individual's self-belief that they are able to complete any set task at any time and are not specific. Task-specific SE beliefs, in the study presented in this paper, will refer to an individual's self-belief that they are able to complete a specific set task presented online. Results of this study will provide knowledge on whether an individual's general SE should be considered in the design of VEI to use within future online learning processes.

\section{General SE and its effect on VEI interpretation}

When considering the influence of VEI on an individual's SE, previous researchers have suggested the consideration of an individual's general SE levels (Bandura, 1994; Bandura \& Wood, 1989). Bandura (1994) outlines how behaviours of those with low general SE, when compared to others with higher levels of SE, differ in four major psychological processes: cognition, selection, motivation and affect. 
With regards to cognitive processes, those with higher levels of general SE find it easier to visualise success scenarios (Bandura, 1994). In contrast, those with lower levels of general SE are more likely to visualise scenarios in which they fail to successfully complete the required task. Selection processes also vary between those with high and low general SE. Those with high general SE have selection processes that allow them to feel more open to trying new tasks (Bandura, 1994). Others with low general SE are more likely to have narrower selection processes which causes them to shy away from any new ventures that they haven't completed successfully in the past. Higher levels of general SE are also linked to higher motivation in goals, allowing greater effort and persistence in the face of difficulties (Bandura, 1994; Bandura \& Wood, 1989). Finally, affective processes differ, with individuals who have higher levels of general SE being able to deal with threats easier than others with low general SE (Bandura, 1994). When faced with a threat, those with low general SE dwell on their coping deficiencies and this can lead to anxiety. This anxiety can lead to them fearing their environment and worrying about the worst outcomes from situations (Bandura \& Wood, 1989).

Considering the behaviours described above, people with varying levels of general SE may be affected differently when presented with VEI. Firstly, due to their inability to visualise success and their susceptibility to be brought down by both negative or positive social comparisons, VEI may be less effective in helping individuals with low general SE. Also, the hesitance and shying away from new ventures that those with low general SE demonstrate could limit how open they are to the positive influence of certain types of VEI. Past experiences could also influence how an individual interprets VEI. If an individual has low general SE because they have experienced failures in related tasks that have been set before, it could be difficult for VEI to increase their SE in being able to attempt completing the task again. This could be due to the prevailing negative memories that gave rise to the individual's current low level of general SE. Finally, individuals with low general SE may be more cautious towards potential threats and less likely to be able to deal with the stress caused by these effectively.

\section{Previous work}

During a literature search, we were unable to find any previous work conducted which examined how the effects of VEI for promoting task-specific SE in OLE's were moderated by general SE. However, we did find previous studies that considered the moderating effect of different types of SE (both general and task-specific) on an individual's interpretation of other types of persuasive messages designed to encourage behavioural changes. The work presented is based upon these types of previous work.

A series of previous studies have supported the key role that SE can play in shaping recipients behavioural responses to health messages with a particular focus on 'framed messages' (Riet, Ruiter, Werrij, \& De Vries, 2008; Riet, Ruiter, Werrij, \& De Vries, 2010a; Riet, Ruiter, Smerecnik, \& De Vries, 2010b; Yang, Chen, \& Wang, 2016). Two equivalent pieces of information can be framed in either a positive or negative way (Block \& Keller, 1995), named 'gain-framed 'or 'loss framed' respectively (Yang et al., 2016). Gain-framing presents the positive outcomes of adherence to the message communicated, for example 'If you reduce your daily sugar intake, you reduce your risk of developing tooth decay'. Loss-framing communicates the negative consequences of non- 
adherence to the message communicated, for example 'If you don't reduce your daily sugar intake, you increase your risk of developing tooth decay :

Riet et al. (2008, 2010a, 2010b) conducted a series of studies looking into the influence that an individual's SE has on how they interpret loss and gain framed health messages. In Riet et al. (2008), researchers studied the effect of message framing on helping individuals to quit smoking. They found that when an individual had high SE in being able to quit, loss-framed messages were more beneficial in encouraging them to take the behavioural steps to stop smoking. For others with lower levels of SE in being able to quit, they found neither loss or gain framed messages to be of any benefit on their behavioural intention to stop smoking. The researchers suggest that individuals with high SE are able to avert the threats presented in loss framed messages more effectively than others with lower SE. This was one of the earliest studies to present the moderating effect that an individual's SE can have regarding the effect of message framing on persuading behavioural change.

Following on from this, Riet et al. (2010a) conducted another study looking into the effect of skin cancer detection messages among university students. The SE described in this study was that of being able to perform a skin cancer self-examination. Once again, this study supported the benefit of using loss framed over gain framed messages for students with higher levels of SE, as these messages were found to increase their intention to perform skin examinations. Also, similar to the earlier study, neither type of framing was found to be of benefit to those with lower SE and their intentions to perform future skin examinations. The researchers suggest that for those with lower $\mathrm{SE}$, the loss framed message presents a great sense of threat. For some this may lead to lower levels of message acceptance, as they are likely to engage in defensive avoidance (e.g. 'That won't happen to me') or message derogation processes (e.g. 'This doesn't apply to me') (Witte, 1992).

In a third study Riet et al. (2010b) explored whether an individual's SE in being able to decrease their salt intake had any influence on the effect of gain and loss framed messages that promoted a low salt diet. The results of this study supported the earlier two studies. As a result, the researchers suggested that loss framed messages may be more effective than gain framed messages in decreasing salt intake, but this is only for individuals that already had a high SE to do so.

Yang et al. (2016) conducted a study investigating the effect of message framing on individuals decisions about undergoing treatment in the form of therapeutic exercise. All of the participants in this study suffered with some form of chronic pain and during the study were shown either loss or gain framed messages about therapeutic exercise. The mediating effect of an individuals SE in being able to complete therapeutic exercise was considered, with the effect on individuals with low and high SE being analysed. Results of this study differed slightly to the earlier studies outlined by Riet et al. $(2008,2010 \mathrm{a}, 2010 \mathrm{~b})$ as they found both low and high SE individuals to be positively influenced by the loss framed message. The researchers put this new result regarding low SE individuals down to self-affirmation. If a low SE individual felt good about themselves generally, this may have reduced the perceived threat of the information and may have lead to them acting in less of a defensive way towards it (Sherman, Nelson, \& Steele, 2000). 
Previous literature has highlighted the differences between the behaviour of those with low and high SE when interpreting certain types of persuasive message. However, there has not been any previous work regarding the interpretation of VEI. Due to the difference in behaviour between those with low and high general SE (Bandura, 1977), it could be assumed that VEI being presented within an OLE may also be interpreted in different ways dependant on the general SE of the individual at the time.

\section{Research purpose and hypotheses}

The effect of an individual's general SE and how it influences the benefit of VEI on task-specific SE within an OLE is an area where little previous work has been conducted. This study will focus on the effects that different forms of VEI have on the task-specific SE beliefs of individuals within an online learning process. Analysis will compare these effects between individuals with low and high levels of general SE. For this study, the following three hypotheses are proposed:

Hypothesis 1: Individuals with low general SE will find VEI less beneficial for increasing their task-specific SE compared to those with high general SE.

Hypothesis 2: Individuals will find VEI demonstrating high levels of success to be more beneficial on their task-specific SE when compared to VEI demonstrating lower levels of success, regardless of their general SE.

Hypothesis 3: Individuals with high general SE will not experience any negative effect on their task-specific SE when exposed to any type of VEI whereas individuals with low general SE will experience negative effects on their task-specific SE as a result of some VEI types.

The results of previous studies have shown individuals with low general SE to find persuasive messages less effective in encouraging behavioural changes (Riet et al., 2008, 2010a, 2010b). Hypothesis 1 predicts, based upon these previous studies, that individuals with low general SE will not find VEI as beneficial on their task-specific SE when compared to others with high general SE.

Hypothesis 2 expects VEI types demonstrating a high level of success to be more beneficial to an individuals task-specific SE than VEI demonstrating lower levels of success. Bandura (1977) suggests that VEI that conveys a clear level of success is more likely to improve an individual's SE more so than information where the success level is unclear or low. Considering this previous literature, this study will investigate the effect of using VEI demonstrating a clear success and whether this is more beneficial on an individuals task-specific SE within a career skills OLE.

Bandura (1994) also outlines characteristics of individuals with high general SE. Hypothesis 3 is based upon these characteristics predicting those with high levels of general SE to be more resilient and therefore less likely to be faulted by negative information, such as VEI demonstrating low levels of success. Because of this, it is expected that no VEI types will have a negative effect on the task-specific SE of individuals with high general SE. In contrast this hypothesis suggests that the task-specific SE of individuals with low general SE may be affected in a negative way by some types of the VEI presented in this study. This is based on characteristics of individuals with low general SE outlined by Bandura (1994) and Bandura and Wood (1989). Those with low 
general SE may experience anxiety or threat when presented with VEI portraying low levels of success, which could have a negative impact on their task-specific SE.

\section{Methodology}

\section{Participants}

Participants were recruited using Amazon mechanical turk. They received a small monetary reward for completing the study. All participants were native english speakers and actively looking for a job at the time of the workshop. One hundred and thirty-six participants took part in the study. Of those that provided demographic information, the sample was 50\% male and 50\% female. Participant age ranged from 20 to 67 ( $M=$ $36, S D=11.4)$. Most participants were in some form of employment: full time $(38 \%)$, part-time $(12 \%)$ or self-employed $(8 \%)$ at the time of completing the study. The full demographic information of the study participants is shown in Table 1.

Before starting the analysis, we grouped participants based upon their general SE beliefs. The data population was split into two halves: low and high general SE groups. Participants were grouped, depending on their general SE score provided in the preworkshop questionnaire. Those in the low SE group had a general SE scale score lower than the total population mean of $31(N=68)$. Participants in the high SE group had a general SE scale score of 31 or above $(N=68)$.

\section{Research design}

We used a between-groups experimental design, with each participant only being shown one type of VEI. In this study, the independent variable was the VEI type that the participant had read. Other variables included in the study include participant general SE, VEI type and benefit of the VEI on participants task-specific SE.

Table 1 Participants demographic details $(n=136)$

\begin{tabular}{lll}
\hline & Frequency & Percent \\
\hline Gender & 68 & $50 \%$ \\
Male & 68 & $50 \%$ \\
Female & & \\
Age & 21 & $16 \%$ \\
$16-25$ & 59 & $46 \%$ \\
$26-35$ & 27 & $21 \%$ \\
$36-45$ & 10 & $8 \%$ \\
$46-55$ & 8 & $6 \%$ \\
$56-65$ & 3 & $2 \%$ \\
$66+$ & & $38 \%$ \\
Career Status & 49 & $12 \%$ \\
Employed - Full Time & 15 & $8 \%$ \\
Employed - Part Time & 10 & $36 \%$ \\
Self - Employed & 46 & $7 \%$ \\
Unemployed & 15 & \\
Student & & \\
\hline
\end{tabular}


In order to analyse the data with reference to Hypothesis 1, significance testing looked for a main effect between a participants level of general SE and the benefit found to their task-specific SE from the information they had read, regardless of what VEI type it was. For Hypothesis 2 analysis, significance testing looked for a main effect between the level of success demonstrated in the VEI (low or high) and the benefit found to a participants task-specific SE after reading the VEI. But further to this, analysis also observed to see if there was an interaction effect between the level of success demonstrated in the VEI and an individuals general SE on how beneficial the VEI is for their task-specific SE. In this case, it is assumed that no significant interaction effect would be present.

Analysis for Hypothesis 3 was conducted in two halves. Firstly, the mean effect of each of the VEI types on the task-specific SE of participants with low and high general SE was calculated. A one sample two-tailed t-test was then conducted to find differences from the mean rating of 3 , 'no effect'. Once these significant differences were found, they were sorted into either significantly positive effects or significantly negative effects on task-specific SE. These significant effects on participants with low and high general SE were then considered when analysing for Hypothesis 3.

\section{VEI design}

There were three types of VEI; a percentage completion statement, a previous answer and a testimonial. For each of these VEI types there were versions demonstrating low and high levels of success, resulting in six different types of VEI altogether. The different types of VEI represented different ways of conveying information about more or less successful completion of tasks by previous participants. All of the VEI forms created were fictitious and were created for the purpose of the study.

The first VEI type conveyed completion directly through a statistic, via a percentage completion statement (See Fig. 1). This statement described the percentage of individuals that had completed the task before them and the percentage that had failed to complete it. The first version of this VEI type stated that a low percentage of participants had completed the workshop successfully $(45 \%)$ and the second version stated a high percentage of previous workshop participants (95\%) had been successful in completing the set task. This type of VEI was chosen because it was considered the most direct way of communicating levels of success to the reader.

The second type of VEI demonstrated more or less successful completion of the task indirectly via an example of a previous participant's answer (See Fig. 2). This type of information had been used in a previous study but was not found to lead to increases in SE beliefs (Newman \& Tuckman, 1997). The first version was an answer that would have scored low according to a mark scheme that was provided earlier on in the workshop. The second version was an answer that would be scored highly according to the

$95 \%$ of participants who started this task completed it, $5 \%$ failed to finish.

Fig. 1 Percentage completion statement (High level of success version) 
Here is an example of how a previous participant completed the task, they scored poorly for their answer:

'An example of this is I solved a tricky problem last week. When it comes to solving problems I believe I habe the right skills to fix them. I would make sure I always use my initiative and that I would solve them quickly and effectively like I did before. '

Fig. 2 Previous participants answer (Low level of success version)

markscheme. This type of VEI conveyed not only success in completing the task but gave information about how to complete the task well through the good and bad examples.

The last type of VEI conveyed success of completion through a testimonial from a previous workshop participant (See Fig. 3), a type of VEI that has also been used in previous studies (Kelly, 2017). The first version was a testimonial in which an individual described how they were unsuccessful in completing the set task. In contrast, the writer of the second testimonial describes how they were able to successfully complete the task set. This type of VEI included the subjective opinions from previous participants about the experience of doing the task.

\section{Measures}

Data was collected using questionnaires at two points during the study: the preworkshop questionnaire and the VEI response questionnaire.

The pre-workshop questionnaire obtained participants demographic data including their age, gender and current employment status. Participants initial levels of general SE were assessed in this questionnaire using the ten item general SE scale (Schwarzer \& Jerusalem, 1995). Evidence of this scales validity has been found in a variety of domains (Grammatopoulou et al., 2014; Mystakidou, Parpa, Tsilika, Galanos, \& Vlahos,

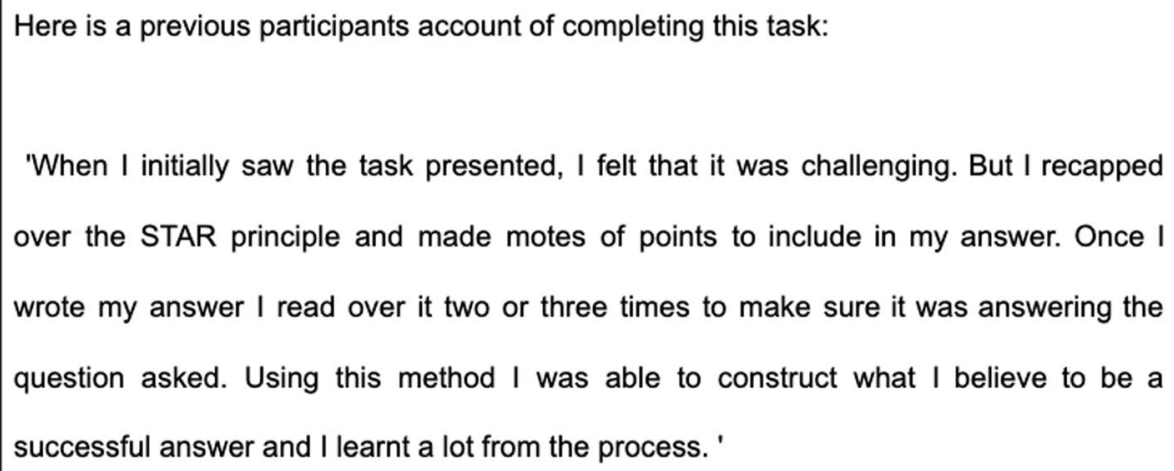

Fig. 3 Participant testimonials (High level of success version) 
2008). In this scale, participants were presented with ten statements and asked to state how true of themselves they feel each statement is. They indicated their answer on a four-point likert scale, ranging from 1 (Not at all true) to 4 (Exactly True). One to four points were awarded for each item based on their scale response, with exactly true having the highest four points awarded and not at all true having the lowest one point awarded. All 10 item points are totalled up to create an overall general SE score, out of a possible 40. During analysis it was assumed that the higher the score, the higher the participants general SE beliefs were at the time.

During the VEI response questionnaire, participants were asked what effect they felt reading the VEI had on their task-specific SE. Participants provided answers on a fivepoint likert scale, ranging from 1 (Strongly decreased) to 5 (Strongly Increased). An open ended question followed directly after this question which asked participants to explain why they felt the VEI had the effect on their task-specific SE that it did. This question would provide qualitative data which would help offer insight into participants reasoning for their earlier answer.

\section{Procedure}

During the study, participants were guided through an online workshop entitled The Career Skills Workshop. The workshop focussed on career skills, providing information on the STAR principle. The STAR principle outlines a structure commonly used to answer competency based questions in job interviews. Competency based questions are where an interviewer asks the interviewee to provide evidence of a particular skill they are looking for in an employee. The workshop was created using the Google forms platform.

Before beginning the workshop, participants were asked to fill in the preworkshop questionnaire which collected their demographic data and starting general SE levels. The workshop started initially by presenting the participant with a number of pages to navigate through. These pages included information about the STAR principle, including an explanation of what it is and how to use it correctly. After reading the workshop material, participants were presented with a small quiz. The quiz contained three multiple choice questions, asking them to recall what they had just read. Anyone who scored 33\% or less in the quiz was discounted from the dataset, as it was concluded that they had not effectively read the information presented to them up to this point.

After completing the quiz, participants were shown a task to complete, the exact task set is outlined in Fig. 4. This task drew upon the skills learnt in the first section of the workshop, asking them to answer an example job interview question using the STAR principle. Participants were shown a mark scheme (see Fig. 5) which outlined the aspects that would make up a high scoring answer. At this point, participants were randomly exposed to one of the six VEI types created. After exposure to the VEI, participants completed the VEI response questionnaire to gauge their reactions to the single VEI they were presented with and the effect it had on their task-specific SE. After this, participants were left to complete the task if they wished. In this study, actual performance on the task was not considered as it was not relevant to the hypotheses posed. 


\section{Your set task will be to write the answer to the following Job Interview question, using the STAR Structure:}

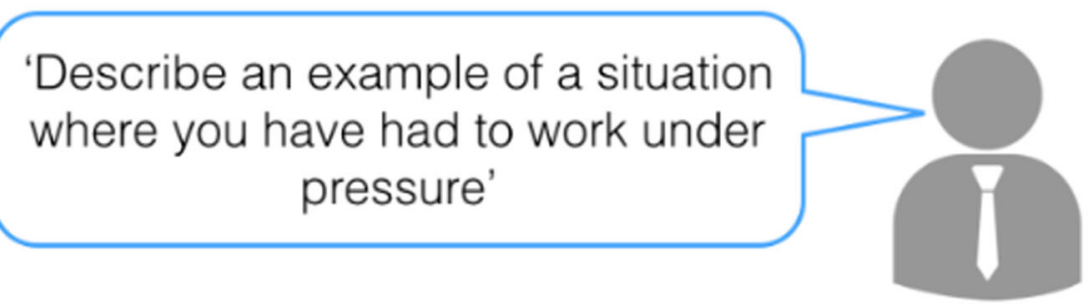

Fig. 4 The set task that participants were asked to complete in the online workshop

\section{Results}

\section{Effect of participants general SE}

The results outlined in this section address Hypothesis 1 directly, considering the effect an individual's general SE has on how much of a boost they experience to their taskspecific SE from reading the VEI presented. Figure 6 presents a comparison of the main effect of participant general SE level on the benefit to their task-specific SE when considering all VEI types combined. Testing found that overall, participants with high general SE found the VEI presented to have a more positive effect on their task-specific SE $(M=3.7, S D=0.80)$ when compared to the low general SE group $(M=3.2, S D=0.81), F$ $(1,124)=15.8, p<.001$.

\section{VEI level of success}

The results in this section address Hypothesis 2, considering the benefit on taskspecific SE of VEI demonstrating low and high levels of success. There was a main effect found of the level of success demonstrated in the VEI presented on the benefit of the information to an individuals task-specific SE. Figure 7 presents a comparison of the effect on task-specific SE between VEI showing low and high levels of success. VEI showing a high level of success was found to have a more positive effect on an individuals task-specific SE $(M=3.6, S D=0.68)$ when compared to VEI showing a lower level of success $(M=3.3, S D=0.95), F(1,124)=6.7, p=.01$. No interaction effect was found between the level of success communicated in the VEI and participants general SE on how beneficial they found the VEI for their task-specific SE, $F(1,124)=0.49, p=.48$.

A three-way ANOVA found a significant two-way interaction between VEI type (percentage statement, previous answer or testimonial) and level of success (low or high) on participants' task-specific SE (Decreased, increased or no effect), $F$ (2, 124) $=9.02, p<.001$.

Figure 8 shows a comparison of the interaction effect on benefit to an individuals task-specific SE of VEI type and level of success demonstrated. Significant differences were found when comparing the effect of low success and high success versions of each of the VEI types. The percentage statement demonstrating the high level of success was found to have a significantly more positive effect on participants' task-specific SE $(M=$ $3.7, S D=0.68$ ) when compared to the percentage statement demonstrating the low 
Your answer will be given a mark between 1 and 10 , with 10 being the highest. This mark will reflect the overall quality of the answer, considering the following aspects:

- Does the answer follow the S.T.A.R. Structure?

- Does it answer the question asked?

- Is the spelling and grammar good?

Fig. 5 Mark scheme shown to participants

level of success $(M=3.1, S D=1.12), t(41)=2.18, p=.03, d=0.64$. The positive testimonial demonstrating a high level of success was also found to be more beneficial for task-specific SE $(M=3.7, S D=0.56)$ when compared to a negative testimonial demonstrating a low level of success $(M=2.9, S D=0.64), t(45)=4.75, p<.001, d=1.32$. However, in the case of the previous answer, the version showing low levels of success was found to have a significantly better effect on task-specific SE $(M=4.0, S D=0.79)$ when compared to the good previous answer demonstrating high levels of success $(M=3.5$, $S D=0.72), t(41)=2.26, p=.03, d=0.66$. This is in contrast to the relationship between levels of success communicated for the other two VEI types presented in the study.

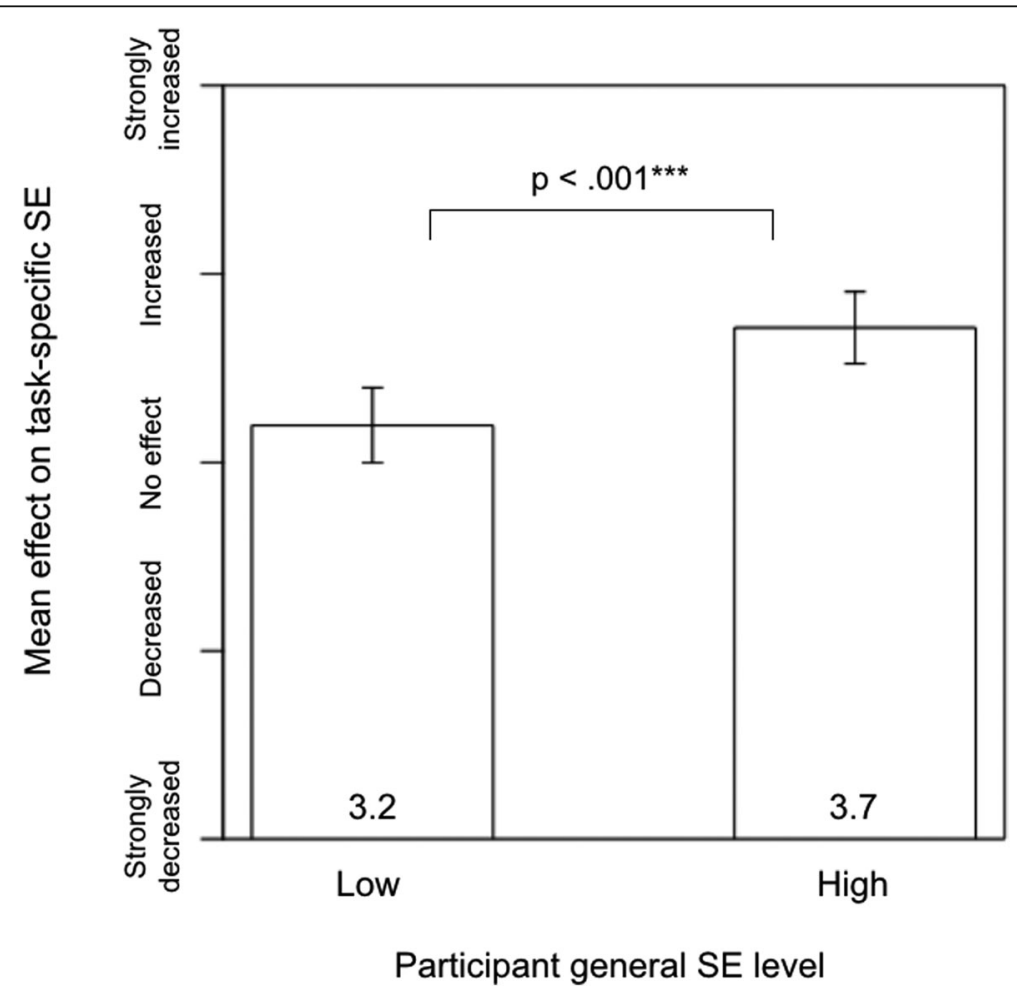

Fig. 6 A comparison of the mean effect of VEI on task-specific SE between participants with low and high levels of general SE 


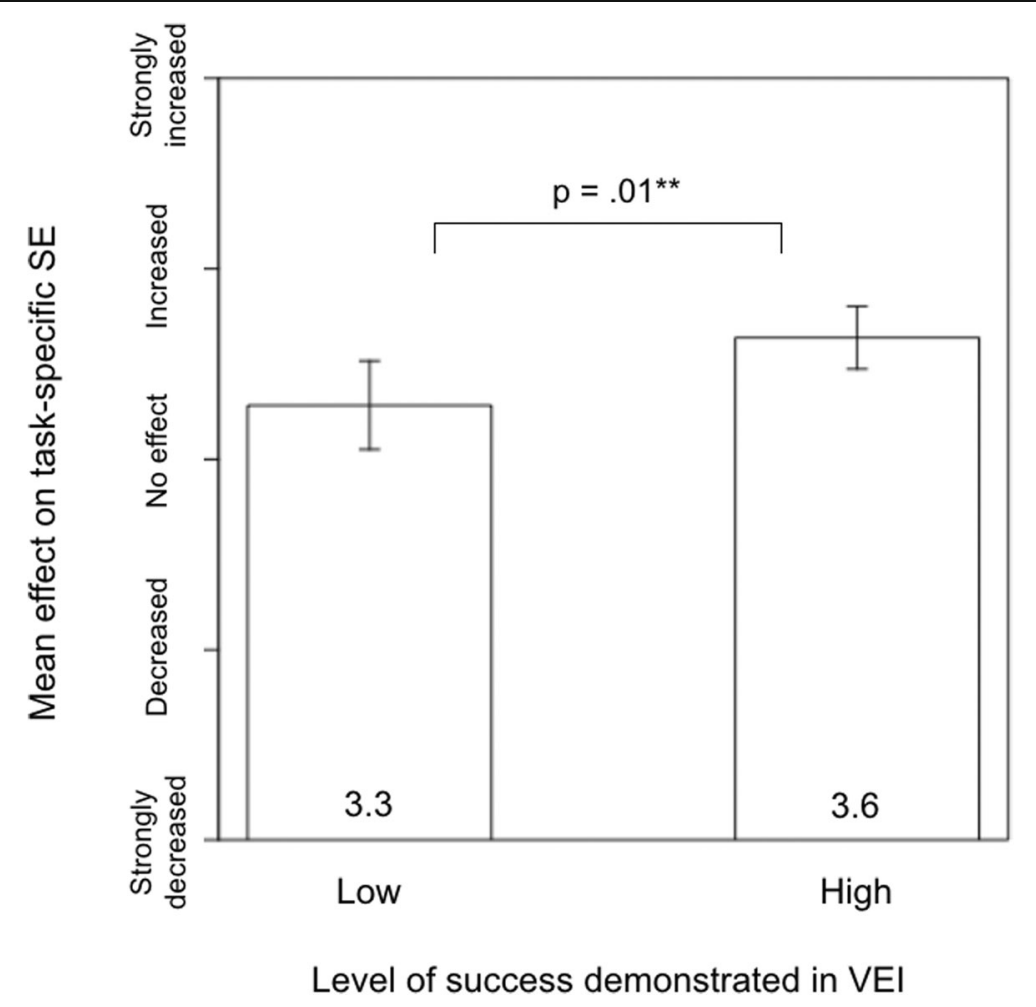

Fig. 7 Comparison of the mean effect on task-specific SE of VEl showing low and high levels of success

When considering the effect in the other direction, a one-way ANOVA considered the benefit of each of the low success VEI types against each other and there was a significant difference found, $F(2,64)=10.9, p<.001$. Post hoc testing found significant differences between the effect on task-specific SE of the low percentage statement and the poor answer. Two sample t-testing showed the bad answer to have a significantly more beneficial effect on task-specific SE $(M=4.0, S D=0.72)$ when compared to the low percentage statement $(M=3.1, S D=1.12), t(33)=3.01, p=.004, d=0.96$. The bad answer was also found to be significantly more beneficial on an individuals task-specific SE when compared to the unsuccessful testimonial $(M=2.9, S D=0.64), t(38)=5.45$, $p<.001, d=1.61$. No differences were found between the effects of the unsuccessful testimonial and the low percentage statement on individuals task-specific SE, $t(28)=$ $0.76, p=.45$. The ANOVA found no significant differences when comparing the benefit of the three successful VEI types against each other, $F(2,66)=0.9, p=.4$.

\section{Potential negative effect of VEI on participants with low vs. high general SE}

To address Hypothesis 3, the presence of significant negative effects on the taskspecific SE of each of the separate VEI types was calculated for those with low and high general SE. A one sample two-tailed t-test was conducted for differences from the mean rating of 3 , 'no effect '.

Figure 9 shows the mean effect of each VEI type on the task-specific SE of individuals with low general SE, with an asterisk highlighting the effects to task-specific SE (both negative and positive) that were found to be significantly different to the known mean 


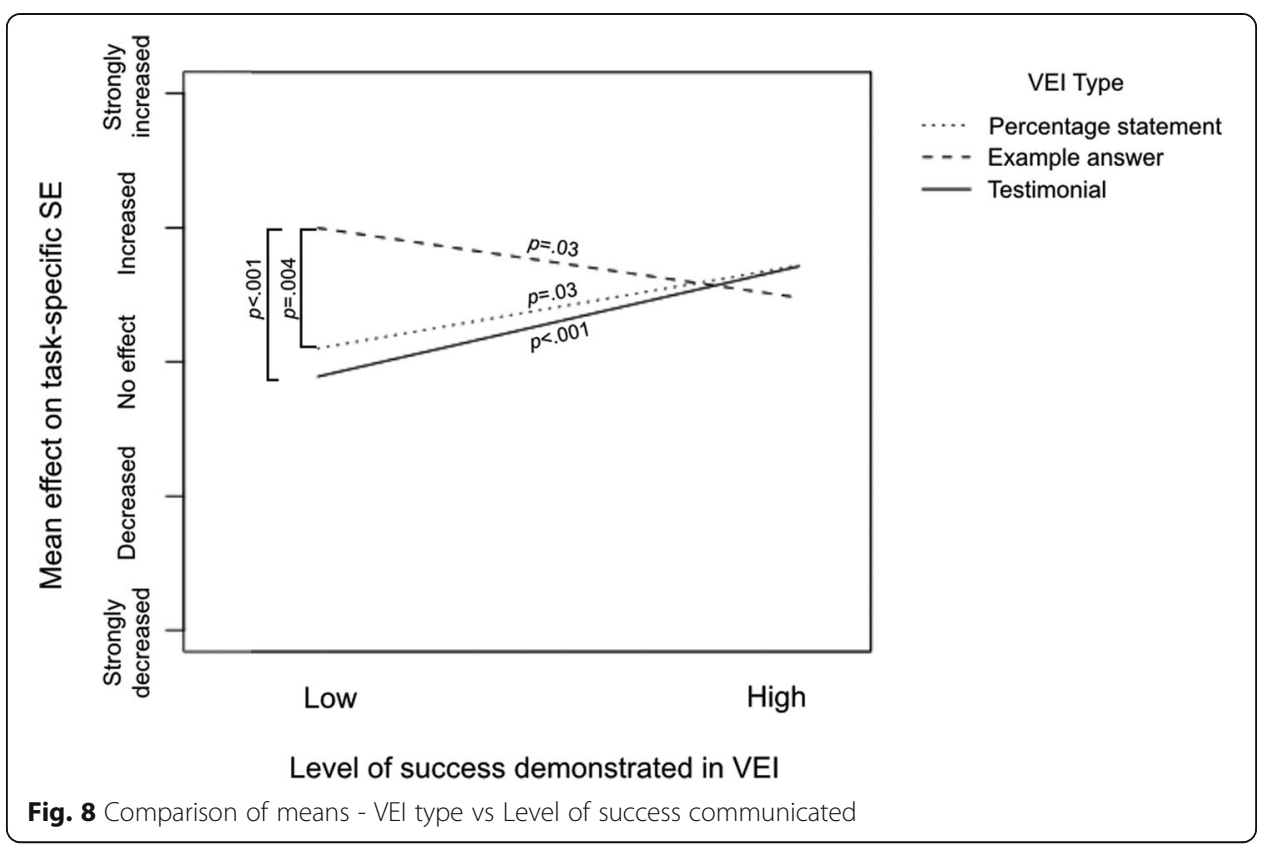

of 3 ('no effect '). In Fig. 9, there is a horizontal dotted line through the Y axis at 'No effect ', representing the boundary between negative and positive effect on task-specific SE. Significant differences to the known mean were found for four of the six VEI types: the high percentage statement, the poor answer, the unsuccessful testimonial and the successful testimonial.

The effect of the high percentage statement $(M=3.3, S D=0.5)$ was found to be significantly different from the known mean, indicating that this type of VEI was significantly beneficial on the task-specific SE of those with low general SE, $t(10)=2.3$, $p=.04, d=0.6$. The poor answer $(M=3.5, S D=0.72)$ was found to be significantly

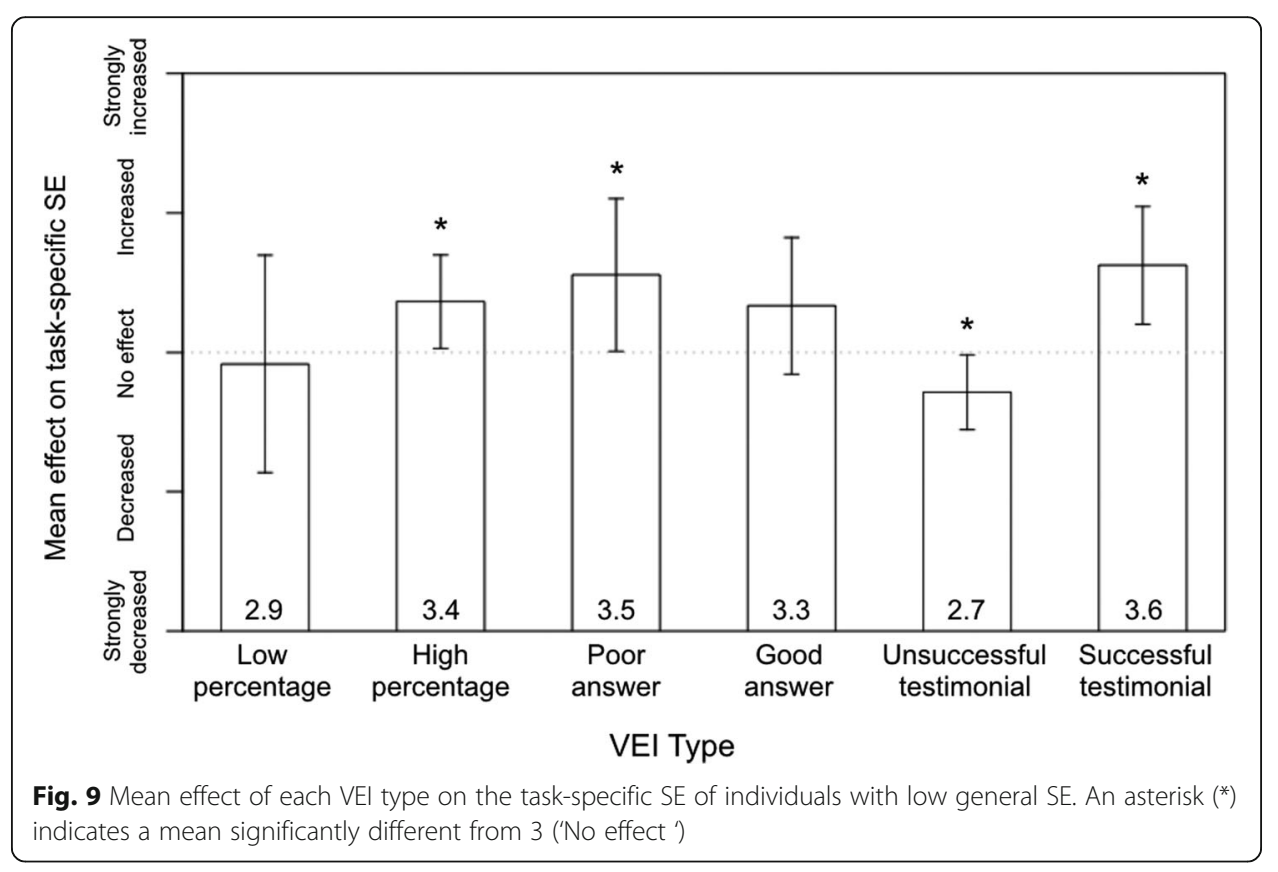


beneficial on task-specific SE of this group also, $t(8)=2.29, p=.05, d=0.69$. Finally, the successful testimonial $(M=3.6, S D=0.52)$ was found to have a significantly positive effect on the task-specific SE of individuals with low general SE, $t(7)=3.42, p=.01$, $d=1.18$. In contrast, the unsuccessful testimonial $(M=2.7, S D=0.47)$ was found to have a significantly negative effect on the task-specific SE of individuals with low general SE, $t(13)=2.28, p=.04, d=0.64$.

Figure 10 shows the mean effect of each VEI type on the task-specific SE of individuals with high general SE, with an asterisk highlighting the effects to task-specific SE (both negative and positive) that were found to be significantly different to the known mean of 3 ('no effect "). There is a horizontal dotted line through the $\mathrm{Y}$ axis at 'No effect ', representing the boundary between negative and positive effect on task-specific SE. One-sample t-tests found significant differences to the known mean for four of the six VEI types (the high percentage information, the poor answer, the good answer and the successful testimonial), with all of them being significantly more beneficial than the known mean.

The effect on task-specific SE from the high percentage information $(M=4, S D=0.68)$ was found to be significantly higher than the known mean $t(13)=5.5, p<.001, d=1.5$. This was also the case when considering the poor answers $(M=4,4, S D=0.5)$ effect on participants task-specific SE, $t(10)=8.9, p<.001, d=2.8$. The good answer $(M=3.6, S D=$ 0.81 ) was found to significantly benefit the task-specific SE when compared to the known mean, $t(10)=2.6, p=.03, d=0.74$. Finally, the last type of VEI found to have a significantly positive effect on task-specific $\mathrm{SE}$ when compared to the known mean was the successful testimonial $(M=3.8, S D=0.60), t(12)=4.62, p<.001, d=1.33$.

\section{Qualitative analysis on influence of VEl types}

Further to the quantitative data outlined above, we collected qualitative data during the study which outlining participants thoughts on how the type of VEI they

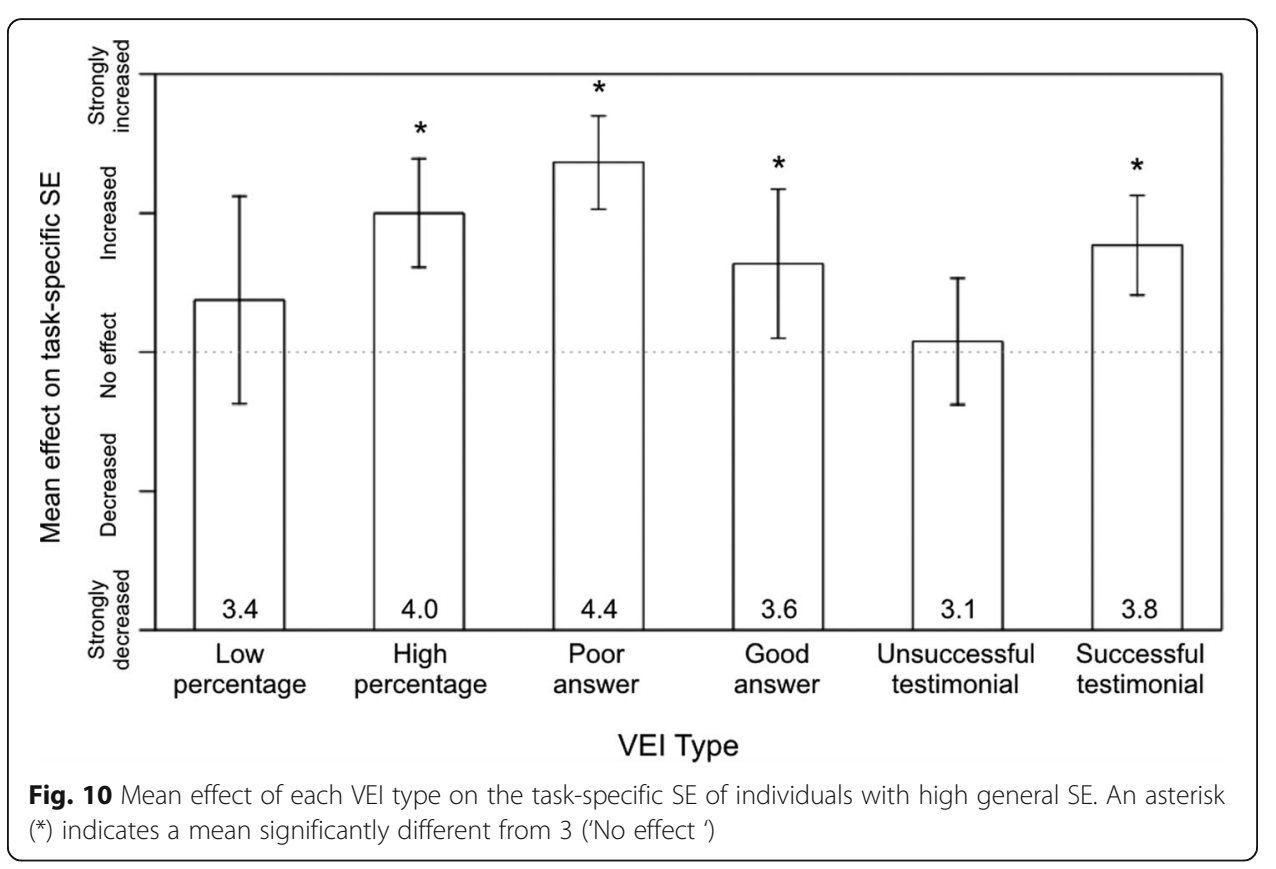


were presented with affected their task-specific SE beliefs. The aim of this analysis was to get a more in depth understanding of possible reasons why each of the VEI types presented affected participants task-specific SE either positively, negatively or not at all. We analysed the qualitative data from the open ended question asked during the study using thematic analysis (Braun \& Clarke, 2006). Common themes mentioned within responses to each VEI type were identified and are outlined below. Themes were sorted by the effect they had on participants task-specific SE (positive, negative or no effect) as well as by whether the participant had low vs. high general SE.

\section{Reasons for positive effect on task-specific SE}

With regards to the positive effects on task-specific SE stated, four main themes were identified which are explained for each VEI form below: self-comparisons, reassurance, reducing task difficulty and gaining of knowledge.

With regards to the high completion statement, participants in both groups made positive self-comparisons after reading the information. They believed if $95 \%$ had been successful in completing the task then they could also be successful. These beliefs had a positive effect on individual 's task-specific SE, 'I feel I'm with the 95\%. If so many can do it, I certainly can'. The fact that so many others had completed it also lead to some participants perceiving the task to be less difficult. This in turn had a positive effect on their task-specific SE, 'Knowing that a very high percentage of participants were able to complete the task lets me know that completing the task must not be that difficult to do'.

Some participants also conducted positive self-comparisons to the bad answer and felt that they could do better, 'The information was not that great and I believe my abilities are beyond what was shown to me'. The bad answer also appeared to reinforce participants awareness in their own ability which had a positive effect on their task-specific SE, 'My awareness of my own ability was reinforced and therefore increased my confidence and motivation to be able to perform effectively and therefore increased my self-belief'. Participants also felt they gained knowledge as the bad answer taught them about what not to do in their answers and this had a positive effect on their task-specific SE, 'It increased my self-belief as I could clearly identify what was lacking in the answer'.

Participants also felt that they gained knowledge from reading the good previous answer, as it provided an example which helped understanding, 'I feel better about how I will handle the task at hand because I now have a better understanding of what is required of me and what form of answer is acceptable. Some participants made positive comparisons between themselves and the testimonial writer. They felt that because the testimonial writer. Had been successful, they could be too, 'Seeing someone else accomplish this goal has made me think that I too can be successful'.

Positive self-comparisons were also made when participants read the unsuccessful testimonial and this was done by both the low and high general SE groups. Participants described how they felt they were more capable than the individual in the testimonial presented, 'My capability is not the same as this persons. What they feel they are capable of doing has no bearing on me'. 
When reading the positive testimonial, participants in the high general SE group felt it increased their task-specific SE because they gained knowledge from it which included tips and examples, 'It gave me a good idea on what to do to solve the task'. Participants in both groups made positive self-comparisons which had a positive effect on their task-specific SE. They felt that if the testimonial writer could do it, then so could they, 'If someone else that was unsure was able to do it that I could too'. Participants in both groups stated that reading the testimonial decreased the perceived task difficulty which had a positive effect on their SE. Overall, participants appeared to find the positive testimonial reassuring and it had a beneficial effect on their task-specific SE, 'I feel like this person boosted my thoughts and ability to do the task at hand?

\section{Reasons for negative effect on task-specific SE}

Two main themes were found within the negative effects the VEI had on participants task-specific SE, worry and negative self-comparisons. With regards to worry, the low percentage statement left many participants in the low general SE group questioning their own beliefs, leading to feelings of uncertainty and worry, 'I feel a little more worried about my ability to complete the task'.

Some participants in the low general SE group made negative self-comparisons to the good answer presented. They felt that they wouldn't be able to write something as good and this lead to a decrease in their perceived task-specific SE, 'A good answer puts me down because I realise that I wouldn't be able to come up with something this easy'. For participants in both the low and high general SE groups, reading the negative testimonial also lead to negative self-comparisons. This resulted in some participants realising they shared the same views as the testimonial writer and this had a negative effect on their task-specific SE, 'Because we share same views and beliefs about the task, I believe I will underperform for the question'.

\section{Reasons for no effect on task-specific SE}

One key theme was highlighted in the comments of those participants of which the VEI had no effect and that was dismissal of the information. This theme was mentioned in participants responses regarding all of the VEI forms presented apart from the good answer. Much of the dismissal was down to participants already having established strong self beliefs, 'I am a very self-confident person, and being shown a percentage of those who complete a task or not does not change my selfconfidence.' Others stated that they are never influenced by external information in cases like this, 'I believe in the talents that I have. I would never let outside forces influence my confidence to such a degree'.

\section{Discussion and conclusion}

Overall, the results of this study have highlighted the mediating effect that an individual's general SE can have on the interpretation and effect of VEI when presented within an OLE. The first hypothesis suggested that based upon previous studies looking into the moderating effect of an individual's general SE on their interpretation of persuasive messages (Riet et al., 2008, 2010a, 2010b), individuals with low general 
SE beliefs would not find VEI to boost their task-specific SE as much as others with high general SE. Results of the study supported this hypothesis. A main effect was found of an individual's general SE level on the boost felt to their task-specific SE from reading the VEI presented. Those with high levels of general SE found reading the VEI to increase their task-specific SE significantly more than participants with low levels of general SE. Results support this hypothesis and show participants with low levels of general SE to find VEI less beneficial for increasing task-specific SE compared to those with high general SE.

The qualitative results highlighted how those with low general SE were less likely to make positive self-comparisons to the VEI they were presented with when compared to the high general SE group. Those in the low general SE group were more likely to make negative self-comparisons to VEI presented, even if it communicated a high level of success. In the case of the good previous answer, some low general SE individuals made negative self-comparisons, feeling that they were not able to create an answer as good as the one they had just read. Results of this study support the mediating role of an individual's general SE in the interpretation of persuasive messages, as suggested in previous studies (Riet et al., 2008, 2010a, 2010b). But this study considered this previous work within the interpretation of persuasive messages in the form of VEI, an information type that had not been considered previously.

The results also supported Hypothesis 2, as a main effect was found of the level of success demonstrated in the VEI on its benefit to an individual's level of task-specific SE. Results describe how study participants perceived the VEI demonstrating a high level of success to be significantly more beneficial to their task-specific SE when compared to VEI communicating low levels of success. Qualitative analysis offered some insight into why participants found VEI demonstrating high levels of success to be beneficial. For some individuals, successful types of VEI provided them with extra information which they felt increased their SE in being able to complete the task. For example, the good answer provided the participants with a guide of how to structure their task answers. Other participants felt successful VEI types encouraged positive self-comparisons. In this process, the participant would compare themselves to the information, which would lead to increases in task-specific SE. In reference to the positive testimonial, some participants felt that if the testimonial writer was able to complete the task successfully, then so could they. VEI demonstrating high levels of success lead to some participants perceiving the task to be less difficult, increasing their task-specific SE. Finally, some participants felt reassured in their own abilities by reading information regarding others successes, this was especially the case for the successful testimonial information.

The qualitative data collected also gave an indication as to why some individuals found VEI types demonstrating low levels of success to decrease their task-specific SE. When reading these VEI types, some individuals would conduct negative selfcomparisons. This process would result in participants feeling that they were not similar at all to the individuals in the VEI presented. Because of this, some participants felt that they would not be able to complete the task. These comparisons would lead to worry, which was found to have a negative effect on individuals task-specific SE.

Where there was a main effect of VEI demonstrating a high level of success to be more beneficial to an individuals task-specific SE than low success VEI types, this was 
caveated by an interaction effect where the bad example answer VEI type was perceived as more beneficial by participants to their task-specific SE than the good example answer. Qualitative data provided by the participants that were presented with the poor answer was useful in understanding why it had a positive effect on some individuals task-specific SE. Some people made positive social comparisons to the poor answer, they felt that they were able to do a lot better and this increased their task-specific SE. For others it was reassuring seeing other people not do that well on the task, and reinforced their beliefs about their own skills and abilities. The characteristics of individuals with high general SE outlined by Bandura (1994) were considered in this hypothesis. As those with high levels of general SE are more resilient and less likely to be faulted by negative information, it was suggested that VEI demonstrating a low level of success would not have a negative impact on their task-specific SE.

Finally, the results presented support Hypothesis 3, as none of the VEI presented in this test had a significantly negative effect on the task-specific SE for individuals with high general SE, not even the VEI demonstrating low levels of success. Qualitative results offered some insight into the thought process of individuals with high general SE when presented with VEI communicating a low level of success. Many individuals in the high general SE group 'dismissed 'any low success VEI they were shown. Some individuals in the high general SE group stated that they already had strong self-beliefs that would not be affected by such information. Others explained how they were never influenced by external information. Overall these results support the resilience that Bandura (1994) suggests those with high general SE have when confronted with negative types of information within an OLE.

The second half of Hypothesis 3 was also supported, as not all VEI presented to those with low general SE had a positive effect on their task-specific SE. A one-sample t-test found the unsuccessful testimonial information to have a significantly negative effect on individuals task-specific SE when compared to the known mean ('No effect '). Qualitative data collected helps to understand why this type of VEI might have a negative effect on the task-specific SE of individuals with low general SE. For some individuals, reading the negative testimonial lead to negative self-comparisons. This was a process in which some participants realised they shared the same views as the person they were presented with. This perceived similarity may lead to the individual believing that because they were similar to the testimonial writer, they too wouldn't be able to complete the set task, leading to the information having a negative effect on their task-specific SE. These results support theory regarding the anxiety and threat an individual with low general SE feels when presented with a VEI demonstrating low levels of success (Bandura, 1994).

Overall, the results have supported all three hypotheses posed in the study, building upon previous work regarding the effect of an individual's general SE on how they interpret persuasive messages. An individual's level of general SE was found to be a mediating factor in the effect different types of VEI had on their task-specific SE within a career skills OLE. Results have shown VEI to be less effective in increasing the task-specific SE of individuals with low general SE beliefs within the context of an OLE. Results suggest a need for VEI to be designed and presented in a way that considers the behaviours of both individuals with high but in particular, low levels of general SE. 
Results support the need for more research to be conducted to find the most effective way of presenting VEI so it increases the task-specific SE of all learners within an OLE, regardless of their general SE. Individuals with low general SE found the unsuccessful testimonial presented in this study to have a negative impact on their task-specific SE, so this type of VEI should be avoided for this group. In contrast, individuals with high general SE didn't find any of the VEI presented to have a negative impact on their taskspecific SE. Results show low general SE individuals to be a more sensitive influenceable group and because of this, care must be taken that their responses to VEI presented are considered differently than those with high SE.

\section{Abbreviations}

OLE: Online learning environment; SE: Self-efficacy; VEl: Vicarious experience information

\section{Acknowledgements}

The authors would like to thank Dr. Antonios Kaniadakis and Claire Revell for their kind help in the dissemination of the workshop.

\section{Authors' contributions}

NW led on writing of the paper with AS also contributing to the writing of the paper. All authors worked on the study design. NW carried out the data analysis, with results being discussed with AS. Both authors read and approved the final manuscript.

\section{Funding}

This work was funded by the Engineering and Physical Sciences Research Council (EPSRC) through the Media and Arts Technology Programme, a Research Councils UK Centre for Doctoral Training (EP/G03723X/1).

\section{Availability of data and materials}

Please contact corresponding author.

\section{Ethics approval and consent to participate}

Before the study was carried out, full ethical approval was obtained from the research ethics committee at Queen Mary University, London. Participants took part voluntarily and were able to leave the study at any time. Whilst taking part in the study, participants were allowed to skip any questions that they didn't feel comfortable in answering. Before the study started participants read an information sheet and were required to give their consent to take part in the study. The information that the participants provided was anonymous and was treated with true confidentiality.

\section{Competing interests}

The authors declare that they have no competing interests.

Received: 4 March 2019 Accepted: 25 June 2019

Published online: 26 July 2019

\section{References}

Ally, M. (2004). Foundations of educational theory for online learning. Theory and Practice of Online Learning, 2, 15-44 Retrieved from http://web.mef.unizg.hr/web/images/pdf/a_online_learning.pdf.

Bandura, A. (1977). Self-efficacy: Toward a unifying theory of behavioral change. Psychological Review, 84(2), 191. https://doi. org/10.1037/0033-295X.84.2.191.

Bandura, A. (1994). Self-efficacy. Hoboken: Wiley.

Bandura, A., \& Wood, R. (1989). Effect of perceived controllability and performance standards on self-regulation of complex decision making. Journal of Personality and Social Psychology, 56(5), 805 Retrieved from https://psycnet. apa.org/buy/1989-27913-001.

Block, L. G., \& Keller, P. A. (1995). When to accentuate the negative: The effects of perceived efficacy and message framing on intentions to perform a health-related behavior. Journal of Marketing Research, 192-203. https://doi.org/10.2307/3152047.

Braun, V., \& Clarke, V. (2006). Using thematic analysis in psychology. Qualitative Research in Psychology, 3(2), 77-101. https:// doi.org/10.1191/1478088706qp063oa.

Gist, M. E., \& Mitchell, T. R. (1992). Self-efficacy: A theoretical analysis of its determinants and malleability. Academy of Management Review, 17(2), 183-211. https://doi.org/10.5465/amr.1992.4279530.

Grammatopoulou, E., Nikolovgenis, N., Skordilis, E., Evangelodimou, A., Haniotou, A., Tsamis, N., \& Spinou, A. (2014). Validity and reliability of general self-efficacy scale in asthma patients. European Respiratory Journal, 44(Suppl 58), P4314 Retrieved from https://erj.ersjournals.com/content/44/Suppl_58/P4314.short.

Kelly, S. L. (2017). First-year students' research challenges: Does watching videos on common struggles affect students' research self-efficacy? Evidence Based Library and Information Practice, 12(4), 158-172. https://doi.org/10.18438/B8QQ28.

Mystakidou, K., Parpa, E., Tsilika, E., Galanos, A., \& Vlahos, L. (2008). General perceived self-efficacy: Validation analysis in Greek cancer patients. Supportive Care in Cancer, 16(12), 1317-1322. https://doi.org/10.1007/s00520-008-0443-z.

Newman, E. J., \& Tuckman, B. W. (1997). The effects of participant modeling on self-efficacy, incentive, productivity, and performance. Journal of Research \& Development in Education Retrieved from http://psycnet.apa.org/record/1997-43779-004. 
Riet, J. V. T., Ruiter, R. A., Smerecnik, C., \& De Vries, H. (2010b). Examining the influence of self-efficacy on message-framing effects: Reducing salt consumption in the general population. Basic and Applied Social Psychology, 32(2), 165-172. https:// doi.org/10.1080/01973531003738338.

Riet, J. V. T., Ruiter, R. A., Werrij, M. Q., \& De Vries, H. (2008). The influence of self-efficacy on the effects of framed health messages. European Journal of Social Psychology, 38(5), 800-809. https://doi.org/10.1002/ejsp.496.

Riet, J. V. T., Ruiter, R. A., Werrij, M. Q., \& De Vries, H. (2010a). Self-efficacy moderates message-framing effects: The case of skin-cancer detection. Psychology and Health, 25(3), 339-349. https://doi.org/10.1080/08870440802530798.

Schunk, D. H. (1987). Peer models and children's behavioral change. Review of Educational Research, 57(2), 149-174.

Schwarzer, R., \& Jerusalem, M. (1995). Generalized self-efficacy scale. In J. Weinman, S. Wright, \& M. Johnston (Eds.), Measures in health psychology: A user's portfolio. Causal and control beliefs, (pp. 35-37). Windsor: NFER-NELSON.

Sherman, D. A., Nelson, L. D., \& Steele, C. M. (2000). Do messages about health risks threaten the self? Increasing the acceptance of threatening health messages via self-affirmation. Personality and Social Psychology Bulletin, 26(9), 10461058. https://doi.org/10.1177/01461672002611003.

Witte, K. (1992). Putting the fear back into fear appeals: The extended parallel process model. Communications Monographs, 59(4), 329-349. https://doi.org/10.1080/03637759209376276.

Yang, D. J., Chen, C. P., \& Wang, C. C. (2016). Would message framing facilitate long-term behavioral change in patients with chronic pain? International Journal of Applied, 6(2) Retrieved from http://www.ijastnet.com/journals/ Vol_6_No_2_June_2016/8.pdf.

\section{Publisher's Note}

Springer Nature remains neutral with regard to jurisdictional claims in published maps and institutional affiliations.

\section{Submit your manuscript to a SpringerOpen ${ }^{\circ}$} journal and benefit from:

- Convenient online submission

- Rigorous peer review

- Open access: articles freely available online

High visibility within the field

Retaining the copyright to your article

Submit your next manuscript at $\boldsymbol{\nabla}$ springeropen.com 